УДК $532.711+66.081 .6$

\title{
Finite Ion Size Effects on Electrolyte Transport in Nanofiltration Membranes
}

\author{
Ilya I. Ryzhkov* \\ Institute of Computational Modelling RAS SB \\ Academgorodok, 50/44, Krasnoyarsk, 660036 \\ Russia \\ Andrey V. Minakov ${ }^{\dagger}$ \\ Institute of Engineering Physics and Radio Electronics \\ Siberian Federal University \\ Svobodny, 79, Krasnoyarsk, 660041
}

Received 25.10.2016, received in revised form 15.11.2016, accepted 10.02.2017

The pressure-driven electrolyte transport through nanofiltration membrane pores with specified wall potential is investigated theoretically. The finite ion size effect is taken into account by introducing an additional term to electrochemical potential. The two-dimensional Navier-Stokes, Poisson, and modified Nernst-Planck equations are solved numerically in a high aspect ratio nanopore connecting two reservoirs with a larger diameter. The calculations are performed for potassium chloride aqueous solution. In the case of point-like ions, the non-physical rise of counter-ion concentration is observed near the pore wall at large applied voltages. When finite ion size is taken in account, the concentration of counter-ions decreases significantly and saturates to the maximum value. It leads to lower osmotic pressure jump and larger magnitude of potential in the pore. The stronger co-ion depletion observed for finite size ions results in the increase of salt rejection, membrane potential, and required pressure drop.

Keywords: conductive membranes, electrolyte transport, nanofiltration, modified Nernst-Planck equation, finite ion size, numerical modelling.

DOI: $10.17516 / 1997-1397-2017-10-2-186-198$

\section{Introduction}

Separation of species with the help of selectively permeable membranes is widely used in various fields of industry including chemical, pharmaceutical, energy, and biotechnology sectors. The removal of ionic species and organic solutes from aqueous solutions can be accomplished with the help of nanofiltration [1]. This pressure-driven membrane process received much attention in the last decades due to its applications in textile, paper, and food industries including water desalination and purification $[2,3]$.

Nanofiltration membranes are characterized by effective pore diameter ranging from one to a few nanometers and applied pressure difference from a few bars up to 10-20 bars. The latter is used to pump the solution through a membrane. The separation of species occurs due to steric and electric effects. In an aqueous solution, most nanofiltration membranes acquire an electric charge due to dissociation of functional groups or adsorption of charged species onto the pore

\footnotetext{
*rii@icm.krasn.ru

$\dagger$ tov-andrey@yandex.ru

(c) Siberian Federal University. All rights reserved
} 
walls. The overlap of electric double-layers in nanometer-sized pores leads to the preferential transport of counter-ions and exclusion of co-ions. If the pore walls are conductive, the wall charge can be altered by applying a prescribed potential to the membrane. In this way, the membrane selectivity characteristics can be externally varied and controlled $[4,5]$.

The understanding of membrane transport mechanisms and prediction of its separation characteristics require the development of adequate mathematical models. There are two fundamental approaches to describing ion transport in charged membranes. In the phenomenological approach based on irreversible thermodynamics, the fluxes of volume, ionic species, and electrical current are related to the driving forces, i.e. the gradients of pressure, species concentration, and electric potential. It provides a relatively simple mathematical description and does not require the knowledge of membrane structure [6]. An alternative approach relates the transport properties of membrane to its structure. Within this approach, one can distinguish heterogeneous models, which consider membrane as a disperse multiphase system, and continuum models describing the transfer within one phase [7]. The latter are based on the Nernst-Planck, Poisson, and Navier-Stokes equations, which are often simplified and approximated.

A charged membrane is usually modelled as an array of identical parallel charged pores. In the homogeneous approach also known as uniform potential (UP) or Teorell-Meyer-Sievers (TMS) model, the radial variation of electric potential and ion concentrations is neglected, and uniform distribution of fixed charged in the pores is assumed [8]. It is valid when the Debye screening length is much larger than the pore radius, and the fixed charge density is sufficiently low. The Donnan-steric partitioning pore model (DSPM) takes into account the finite ion size by introducing diffusive and convective hindrance factors. The fitting of experimental rejection data to this model allowed obtaining such membrane parameters as pore radius, volume charge density, and effective membrane thickness [9]. The effect of concentration polarization on salt rejection was investigated in [10] by coupling the DSPM model with convection-diffusion equations in the boundary layer adjacent to the membrane. The influence of dielectric effects on ion rejection in nanofiltration membranes was actively studied in recent decades [11]. These effects are related to the interaction of ions with polarized interface between membrane and solution due to the difference in their dielectric constants and lowering of solution dielectric constant inside nanopores.

The radial variation of potential and ion concentrations in the pore as well as the impact of electroosmotic flow on ion transfer are taken into account in the space-charge (SC) model, which was originally developed in [12]. Experimental verification of this model was performed by comparing the theoretical predictions for streaming potential, pore conductivity, and diffusion potential with the measured data [13]. A good agreement for monovalent ions was found. Detailed comparison between results obtained from SC and TMS models [14] showed a good agreement for pores with small radius (less than $2 \mathrm{~nm}$ ) and low surface charge density. Otherwise, a strong overestimation of rejection by the TMS model in comparison with the SC model was found. These conclusions were later confirmed by comparing the results from Uniform potential model with full 2D numerical modelling [15]. Different contributions of diffusion and migration fluxes to ion rejection were observed for cases of constant surface potential / charge density. The SC model was revisited in [16] by using the flux-force formalism with Onsager symmetry properties, which allowed a significant simplification of working formulas.

In the frame of Poisson-Boltzmann approach employed in uniform potential and space-charge models, the assumption of point-like ions is made. However, it is known to strongly overestimate ionic concentration near highly charged surfaces, especially for solutions with multivalent 
ions [17]. To overcome this limitation, the modified Poisson-Boltzmann approach was proposed by taking into account steric effects [18]. The corresponding equations can be derived by the variation of free energy, where the steric correction is included in the entropic contribution [17]. Based on this approach, the impact of steric effects on the dynamics of electrolytes near a surface with high applied voltage was studied in $[19,20]$. It was found that the salt adsorption by the double layers was greatly reduced due to saturation of ionic concentration near the surface. The induced-charge electrokinetics at large applied voltages in concentrated solutions was reviewed in [21]. It was shown in [22] that steric correction leads to higher current efficiency in charged membrane with narrow pores. The study of electrokinetic flow in nanoporous membranes driven by concentration gradients revealed that ion size effects decrease the average ionic concentration in the pore solution, reduce the absolute values of average solute flux density and solution velocity, and increase the membrane potential [23].

In this paper, we first investigate the impact of finite ion size on the pressure-driven electrolyte transport in nanofiltration membranes. The system is described by two-dimensional NavierStokes, Poisson, and Nernst-Planck equations. The latter is modified to take into account steric effects, which allows considering a wide range of voltages applied to the conductive pore wall. The equations are solved in a high aspect ratio nanopore connecting two reservoirs with much larger diameter. Detailed comparison between results for point-like and finite size ions is performed.

\section{Mathematical model}

\subsection{Modified Nernst-Planck equation}

Consider a binary symmetric electrolyte with concentrations of positive and negative ions denoted by $C_{+}$and $C_{-}$, respectively. The electrochemical potentials of ions (in Joule/mole), which take into account the steric effect, can be written in the form [17,20,24]

$$
\mu_{ \pm}=\mu_{ \pm}^{0}+R T \ln \left(N_{A} a_{ \pm}^{3} C_{ \pm}\right) \pm F \Phi-R T \ln \left(1-N_{A} a_{+}^{3} C_{+}-N_{A} a_{-}^{3} C_{-}\right) .
$$

Here $\mu_{ \pm}^{0}$ are the standard chemical potentials, $R$ is the ideal gas constant, $T$ is the temperature, $F$ is the Faraday constant, $\Phi$ is the electric potential, $N_{A}$ is the Avogadro number, and $a_{ \pm}$are the diameters of solvated ions. The derivation of (1) is based on the free energy functional $\mathcal{F}$, which includes the electrostatic energy contribution (self-energy of electric field and electrostatic energies of ions) and entropic contribution (entropies of positive and negative ions as well as solvent molecules) $[17,20,25]$. The requirement of zero functional derivatives of $\mathcal{F}$ with respect to $C_{ \pm}$leads to equations (1), where the last term accounts for ion size effect and penalizes large ionic concentrations.

The ion fluxes are driven by the chemical potential gradients [26], so one can write

$$
J_{ \pm}=-C_{ \pm} \frac{D_{ \pm}}{R T} \nabla \mu_{ \pm}=-D_{ \pm} \nabla C_{ \pm} \mp D_{ \pm} C_{ \pm} \frac{F}{R T} \nabla \Phi-D_{ \pm} C_{ \pm} \frac{N_{A}\left(a_{+}^{3} \nabla C_{+}+a_{-}^{3} \nabla C_{-}\right)}{1-N_{A}\left(a_{+}^{3} C_{+}+a_{-}^{3} C_{-}\right)},
$$

where $D_{ \pm}$are the ions diffusion coefficients.

The first and second terms in the right-hand side of (2) describe the ion transport by diffusion and migration in electric field, respectively. The third terms corresponds to an additional flux, which is directed against ion concentration gradients and becomes very large when the volume fraction of solvated ions $N_{A}\left(a_{+}^{3} C_{+}+a_{-}^{3} C_{-}\right)$approaches unity (a simple cubic packing of ions with effective diameters $a_{ \pm}$is assumed). It prevents the non-physical increase of concentration 
observed in the Poisson-Boltzmann approach [17]. The expression for ion fluxes (2) will be referred to as modified Nernst-Planck equation. It reduces to classical Nernst-Planck equation when the third term accounting to ion size effect is absent.

\subsection{Problem statement}

To study the electrolyte transport through membrane, we consider a single cylindrical nanopore of length $L_{p}$ and radius $R_{p}$, which connects two reservoirs of length $L_{r}$ and radius $R_{r}$. The axisymmetric geometry of the system is shown in Fig. 1. In this work, it is assumed that the pore walls are conductive, and a constant potential $\varphi_{s}$ is specified on them. The electrolyte with fixed entrance concentration $C^{L}$ is pumped through the pore with the volume flux $J_{V}$, which is equivalent to the radially averaged velocity.

The stationary flow and ion transport are described by the Navier-Stokes, Nernst-Plank, and Poisson equations $[15,16,21]$. We introduce dimensionless variables by taking the characteristic scales of length $L$ (to be specified later), velocity $J_{V}$, pressure $\rho J_{V}^{2}$, concentration $C^{L}$, and potential $R T / F$. The dimensionless governing equations are written in the form

$$
\begin{gathered}
(\boldsymbol{U} \cdot \nabla) \boldsymbol{U}=-\nabla P+\frac{1}{\mathrm{Re}} \nabla^{2} \boldsymbol{U}-\Lambda\left(C_{+}-C_{-}\right) \nabla \Phi, \quad \nabla \cdot \boldsymbol{U}=0, \\
\operatorname{Pe} \boldsymbol{U} \cdot \nabla C_{+}=-\nabla \cdot J_{+}, \quad \operatorname{Pe} \boldsymbol{U} \cdot \nabla C_{-}=-\nabla \cdot D J_{-} \\
\nabla^{2} \Phi=\frac{1}{2 \lambda^{2}}\left(C_{+}-C_{-}\right),
\end{gathered}
$$

where the dimensionless ion fluxes are given by

$$
J_{ \pm}=-\nabla C_{ \pm} \mp C_{ \pm} \nabla \Phi-C_{ \pm} \frac{\nu_{+} \nabla C_{+}+\nu_{-} \nabla C_{-}}{1-\nu_{+} C_{+}-\nu_{-} C_{-}} .
$$

In equations (3)-(5), $\boldsymbol{U}$ is the velocity vector and $P$ is the pressure. For axisymmetric problem, the velocity is written as $\boldsymbol{U}=(U, V)$, where $U$ and $V$ are the radial and axial velocity components, respectively. The equations are solved in cylindrical coordinates $(r, z)$.

The dimensionless parameters of the problem are written as

$$
\begin{aligned}
& \mathrm{Re}=\frac{\rho J_{V} L}{\mu}, \quad \mathrm{Pe}=\frac{J_{V} L}{D_{+}}, \quad \Lambda=\frac{R T C^{L}}{\rho J_{V}^{2}}, \quad D=\frac{D_{-}}{D_{+}}, \\
& \nu_{ \pm}=C^{L} N_{A} a_{ \pm}^{3}, \quad \lambda=\frac{1}{L} \sqrt{\frac{\varepsilon \varepsilon_{0} R T}{2 C^{L} F^{2}}}, \quad \varphi_{s}^{\prime}=\frac{F \varphi_{s}}{R T}, \quad J_{V}^{\prime}=\frac{R_{p}^{2}}{R_{r}^{2}},
\end{aligned}
$$

where Re is the Reynolds number, Pe is Peclet number, $\Lambda$ is the parameter, which characterizes the electric (Coulomb) force, $D$ is the ratio of diffusion coefficients, $\nu_{ \pm}$are the volume fractions of ions at the inlet, $\lambda$ is the dimensionless Debye length, $\varphi_{s}^{\prime}$ is the dimensionless wall potential, and $J_{V}^{\prime}$ is the dimensionless velocity at the inlet. It is chosen from the condition of equal flow rates in the reservoirs and nanopore. In formulas (7), $\rho$ is the density of ionic solution, $\varepsilon$ is its relative permittivity, and $\varepsilon_{0}$ is the vacuum permittivity. The case of point-like ions is recovered by taking $\nu_{ \pm}=0$.

The boundary conditions are summarized in Tab. 1. An outward unit normal to the nanopore wall is denoted by $\boldsymbol{n}$. At the inlet $A_{1}$, the constant dimensionless axial velocity $J_{V}^{\prime}$, zero potential, and constant dimensionless concentration are specified. At the outlet $B_{1}$, we impose zero pressure, the absence of potential gradient, and zero fluxes of ions due to diffusion and 
migration (the transport of ions occurs by convection only). The same conditions are specified on the reservoir walls $A_{2}, A_{3}, B_{2}, B_{3}$ except zero pressure requirement, which is replaced by the no-slip condition. The latter is also specified on the nanopore wall $C$ together with zero diffusion and migration fluxes. In addition, we impose constant potential on the nanopore wall. The wall potential is set relative to the bulk solution at the inlet $A_{1}$, where zero potential is specified.

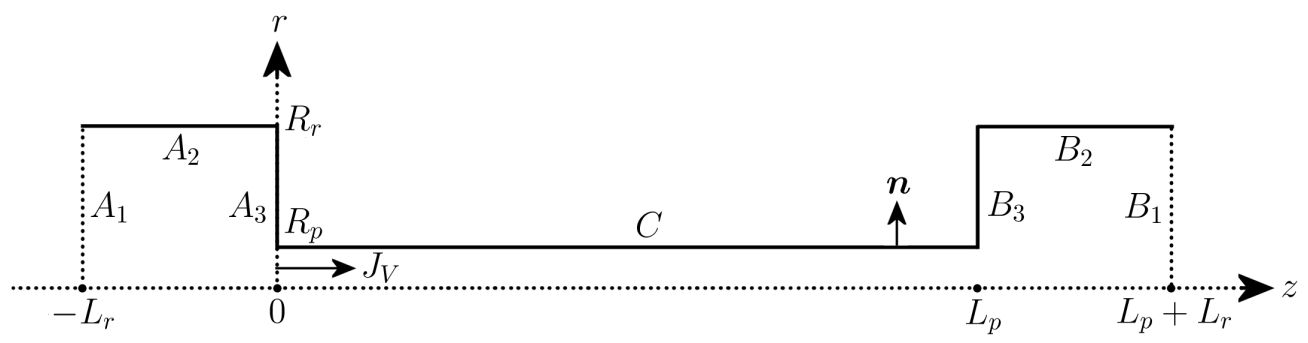

Fig. 1. The geometry of the nanopore and reservoirs

Table 1 . The boundary conditions

\begin{tabular}{ccccc}
\hline \hline $\begin{array}{c}\text { Boundary } \\
\text { segment }\end{array}$ & $\begin{array}{c}\text { Navier-Stokes } \\
\text { equations }\end{array}$ & $\begin{array}{c}\text { Poisson } \\
\text { equation }\end{array}$ & $\begin{array}{c}\text { Nernst-Planck } \\
\text { equations }\end{array}$ & $\begin{array}{c}\text { Nernst-Planck eqns. } \\
\text { (Slotboom variables) }\end{array}$ \\
\hline$A_{1}$ & $U=0, V=J_{V}^{\prime}$ & $\Phi=0$ & $C_{ \pm}=1$ & $\widetilde{C}_{ \pm}=\left(1-\nu_{+}-\nu_{-}\right)^{-1}$ \\
$B_{1}$ & $P=0$ & $\nabla \Phi \cdot \boldsymbol{n}=0$ & $J_{ \pm} \cdot \boldsymbol{n}=0$ & $\nabla \widetilde{C}_{ \pm} \cdot \boldsymbol{n}=0$ \\
$A_{2,3}, B_{2,3}$ & $U=V=0$ & $\nabla \Phi \cdot \boldsymbol{n}=0$ & $J_{ \pm} \cdot \boldsymbol{n}=0$ & $\nabla \widetilde{C}_{ \pm} \cdot \boldsymbol{n}=0$ \\
$C$ & $U=V=0$ & $\Phi=\varphi_{s}^{\prime}$ & $J_{ \pm} \cdot \boldsymbol{n}=0$ & $\nabla \widetilde{C}_{ \pm} \cdot \boldsymbol{n}=0$ \\
\hline \hline
\end{tabular}

\subsection{Slotboom variables}

In this work, we propose an alternative formulation of governing equations by introducing the Slotboom variables $[25,27]$ (transformed concentrations) according to

$$
\widetilde{C}_{ \pm}=C_{ \pm} \exp \left( \pm \Phi-\ln \left(1-\nu_{+} C_{+}-\nu_{-} C_{-}\right)\right)=\frac{C_{ \pm} e^{ \pm \Phi}}{1-\nu_{+} C_{+}-\nu_{-} C_{-}} .
$$

The original concentrations can be restored from the transformed ones by the formulas

$$
C_{ \pm}=\rho_{ \pm} \widetilde{C}_{ \pm}, \quad \rho_{+}=\frac{1}{e^{\Phi}+\nu_{+} \widetilde{C}_{+}+e^{2 \Phi} \nu_{-} \widetilde{C}_{-}}, \quad \rho_{-}=\frac{1}{e^{-\Phi}+e^{-2 \Phi} \nu_{+} \widetilde{C}_{+}+\nu_{-} \widetilde{C}_{-}} .
$$

The governing equations (3)-(5) are rewritten in Slotboom variables as follows

$$
\begin{gathered}
(\boldsymbol{U} \cdot \nabla) \boldsymbol{U}=-\nabla P+\frac{1}{\operatorname{Re}} \nabla^{2} \boldsymbol{U}-\Lambda\left(\rho_{+} \widetilde{C}_{+}-\rho_{-} \widetilde{C}_{-}\right) \nabla \Phi, \quad \nabla \cdot \boldsymbol{U}=0, \\
\nabla \cdot\left(\rho_{+}\left(\operatorname{Pe} \boldsymbol{U} \widetilde{C}_{+}-\nabla \widetilde{C}_{+}\right)\right)=0, \quad \nabla \cdot\left(\rho_{-}\left(\operatorname{Pe} \boldsymbol{U} \widetilde{C}_{-}-D \nabla \widetilde{C}_{-}\right)\right)=0, \\
\nabla^{2} \Phi=\frac{1}{2 \lambda^{2}}\left(\rho_{+} \widetilde{C}_{+}-\rho_{-} \widetilde{C}_{-}\right) .
\end{gathered}
$$


After the transformation, the boundary conditions for Navier-Stokes and Poisson equations remain the same, while the conditions for the Nernst-Planck equations are transformed into a simpler form, which is given in the last column of Tab. 1. In Slotboom variables, the equations for ion concentrations (4) are also simplified and take the form of (11). It will be shown in Sec. 3 that using the Slotboom variables allows decreasing the computational time as well.

Note that the flow in nanopores corresponds to low Reynolds numbers, so the convective term in equations (3) and (10) can be neglected. However, we have retained it for completeness.

\subsection{Physical parameters}

The calculations have been performed for potassium chloride aqueous solution, which fully disassociates into $\mathrm{K}^{+}$and $\mathrm{Cl}^{-}$ions. The parameters used are as follows: $T=298.15 \mathrm{~K}, R=$ $8.314 \mathrm{~J} /(\mathrm{mol} \mathrm{K}), \quad F=96485 \mathrm{C} / \mathrm{mol}, \quad \varepsilon=78.49, \quad \varepsilon_{0}=8.854 \cdot 10^{-12} \mathrm{~F} / \mathrm{m}, \rho=997 \mathrm{~kg} / \mathrm{m}^{3}$, $\mu=0.888 \cdot 10^{-3} \mathrm{~Pa} \cdot \mathrm{s}, D_{+}=1.957 \cdot 10^{-9} \mathrm{~m}^{2} / \mathrm{s}, D_{-}=2.032 \cdot 10^{-9} \mathrm{~m}^{2} / \mathrm{s}, a_{+}=a_{-}=0.66 \cdot 10^{-9} \mathrm{~m}$, $L=10^{-9} \mathrm{~m}$. The size of reservoirs is fixed to $R_{r}=25 \mathrm{~nm}, L_{r}=100 \mathrm{~nm}$, while the nanopore dimensions are $R_{p}=2 \mathrm{~nm}, L_{p}=2000 \mathrm{~nm}$. The inlet concentration is taken as $C^{L}=10$ $\mathrm{mol} / \mathrm{m}^{3}$ and the inlet velocity is $J_{V}=100 \mu \mathrm{m} / \mathrm{s}$. The wall potential varies in the range $\varphi_{s}=$ $-0.05 \ldots-0.30 \mathrm{~V}$. The chosen dimensional parameters correspond to the following values of dimensionless parameters defined by $(7): \operatorname{Re}=1.123 \cdot 10^{-5}, \mathrm{Pe}=5.110 \cdot 10^{-5}, \Lambda=2.486 \cdot 10^{9}$, $D=1.038, \nu_{ \pm}=1.73 \cdot 10^{-3}, \lambda=3.042, J_{V}^{\prime}=6.4 \cdot 10^{-3}$. The range of dimensionless wall potential is $\varphi_{s}^{\prime}=-1.946 \ldots-11.677$.

\section{Numerical implementation}

The governing equations were integrated numerically with a help of ANSYS Fluent 14.5 (Academic version). The stationary problem was solved iteratively by the SIMPLEC scheme in finite volume formulation [28]. The characteristics of computational mesh were chosen in such a way as to ensure that further refinement did not influence the solution quality. For the typical case of $R_{p}=2 \mathrm{~nm}, L_{p}=2000 \mathrm{~nm}, R_{r}=25 \mathrm{~nm}, L_{r}=100 \mathrm{~nm}$, the number of nodes in radial and axial directions was 20 and 3000 for the nanopore, and 70 and 120 for each reservoir, respectively. The mesh had clustering in the axial direction near the nanopore entrance and exit as well as in the radial direction in the reservoirs. In the nanopore, the mesh was radially uniform.

We have performed a comparison of convergence between formulations in Primitive variables (3)-(5) and Slotboom variables (10)-(12). It was found that the use of Slotboom variables allows to decrease the required number of iterations by 2 times. The total number of iterations in this case was around 15000 . The calculations were performed with the serial processing option (i.e. on a single core). It was found that the use of parallel computations significantly increases the number of iterations required for convergence. In ANSYS Fluent, the parallel computations are realized on the level of linear algebraic equations solver. The decomposition of computational domain requires solution matching, which apparently decreases the convergence rate.

\section{Results and discussion}

To describe the electrolyte transport in the nanopore, we need to introduce some characteristics in addition to those already defined in Sec. 2. The rejection (i.e. the fraction of ions, which 
is retained by the membrane) is defined as

$$
\mathrm{R}=1-\frac{C^{R}}{C^{L}} .
$$

For the considered problem, $C^{L}=C_{ \pm}(0)$ and $C^{R}=C_{ \pm}\left(L_{p}+L_{r}\right)$. To study the axial variations of all quantities, the cross-sectionally averaged variables are introduced by

$$
\bar{\Phi}=\frac{1}{\pi R_{p}^{2}} \int_{0}^{2 \pi} \int_{0}^{R_{p}} \Phi r d r d \varphi, \quad \bar{C}_{ \pm}=\frac{1}{\pi R_{p}^{2}} \int_{0}^{2 \pi} \int_{0}^{R_{p}} C_{ \pm} r d r d \varphi, \quad \bar{P}=\frac{1}{\pi R_{p}^{2}} \int_{0}^{2 \pi} \int_{0}^{R_{p}} P r d r d \varphi .
$$

where $\Phi, C_{ \pm}, P$ are the dimensional potential, ion concentrations, and pressure, respectively. In what follows, we will also need the membrane potential $\Delta \bar{\Phi}$ and the applied pressure drop $\Delta \bar{P}$, which are calculated as

$$
\Delta \bar{\Phi}=\bar{\Phi}\left(L_{p}+L_{r}\right)-\bar{\Phi}(0)=\bar{\Phi}\left(L_{p}+L_{r}\right), \quad \Delta \bar{P}=\bar{P}(0)-\bar{P}\left(L_{p}+L_{r}\right)=\bar{P}(0) .
$$

Let us start the discussion of results with the case of wall potential $\varphi_{s}=-0.20 \mathrm{~V}$. The profiles of potential, pressure, ion concentrations and solution velocity at the central crosssection $z=L_{p} / 2$ are shown in Fig. 2 for point-like and finite size ions. The electric potential increases in magnitude towards the pore wall, and causes co-ion $\left(\mathrm{Cl}^{-}\right)$depletion and counterion $\left(\mathrm{K}^{+}\right)$enrichment in the pore. For point-like ions, the latter is much larger near the wall and slightly smaller in rest part of the pore in comparison with the finite size ions, see Fig. 2 (c).
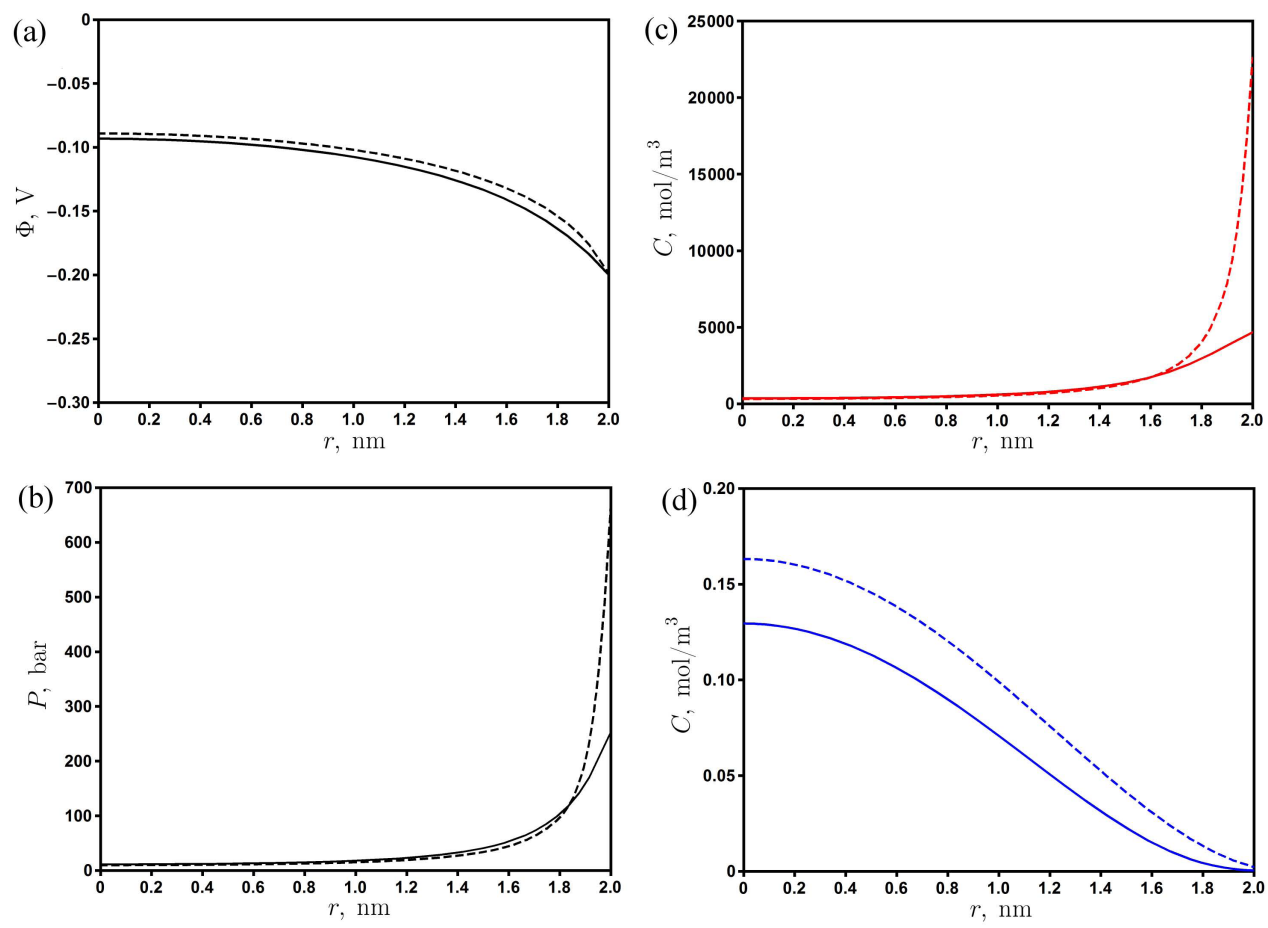

Fig. 2. The profiles of potential (a), pressure (b), concentrations of positive (c) and negative (d) ions in the cross-section $z=L_{p} / 2$ for $\varphi_{s}=-0.20 \mathrm{~V}$. Finite size ions (solid line), point-like ions (dashed line) 
The same is true for the pressure profile in Fig. 2(b). The pressure gradient near the wall compensates the electric force (the last term in equation (3)), which is directed towards the wall. The counter-ion concentration near the wall is non-physically large for point-like ions and far exceeds the limit set by finite ion size (see also Fig. 4 (b) below). The concentration of co-ions is smaller when the steric effect is taken into account. These results are in line with previous calculations in the absence of pressure-driven flow [23]. The calculations show that for small potentials $\left(\left|\varphi_{s}\right|<0.1 \mathrm{~V}\right)$ the curves for point-like and finite size ions become much closer.

The distributions of averaged potential and ion concentrations for $\varphi_{s}=-0.20 \mathrm{~V}$ are shown in Fig. 3. The steric effect leads to smaller counter-ion concentration and, consequently, smaller osmotic pressure jumps at the pore entrance and exit. These jumps balance the electric force, which develops at the interfacial regions, where non-zero net charge is subjected to a large electric field. The pressure gradient inside the pore is mainly determined by the specified volume flux $J_{V}$.
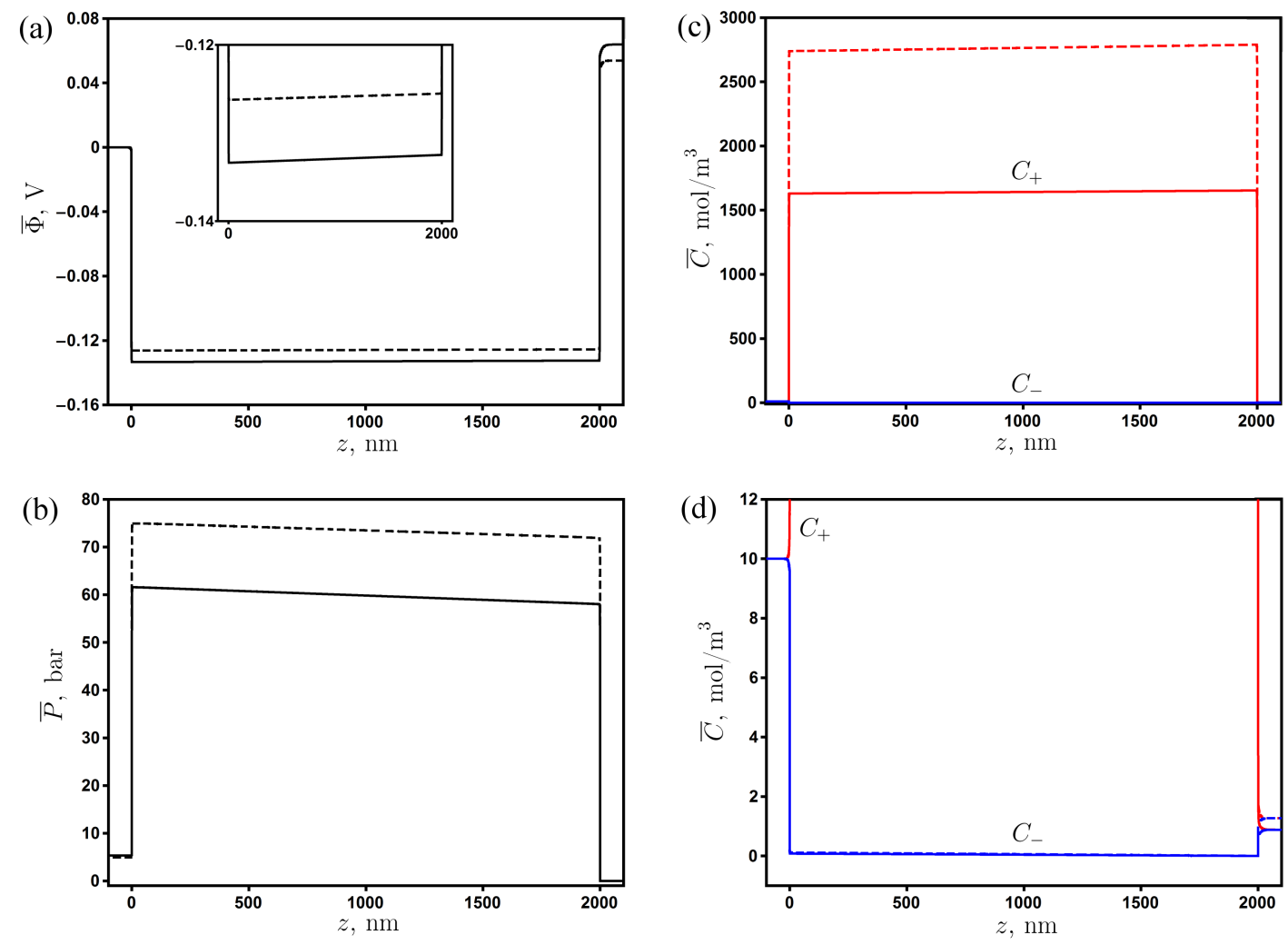

Fig. 3. The profiles of averaged potential (a), pressure (b), concentrations of positive (c) and negative (d) ions for $\varphi_{s}=-0.20 \mathrm{~V}$. Finite size ions (solid line), point-like ions (dashed line)

The decrease of average counter-ion concentration when steric effect is accounted for induces a slight increase of potential in magnitude, see Figs. 2 (a) and 3 (a). Detailed analysis shows the development of small axial potential gradient directed to the pore exit (it shown at the inset to Fig. 3(a)). The corresponding axial electric force acts on the non-compensated charge in the direction of nanopore entrance, and its magnitude is stronger near the nanopore wall. It leads to the deviation of velocity profile from the parabolic one given by $V=2 J_{V}\left(1-\left(r / R_{p}\right)^{2}\right)$. Near 
the wall, the flow is slightly slowed down, while near the center of the channel it is enhanced to provide the same averaged volume flux $J_{V}$.

The stronger depletion of co-ions in case of finite ion size results in the smaller value of electrolyte concentration at the outlet in comparison with point-like ions, so the rejection of salt is enhanced. As a result, the membrane potential $\Delta \bar{\Phi}$, which is mainly determined by the Donnan potential jumps at the pore entrance and exit, increases (Fig. 3 (a)). The mechanism of rejection for constant wall potential is related to balancing of counter-ions convective flux by the opposite migration and diffusion fluxes. In this case, the total flux of counter-ions becomes equal to that of co-ions, which is very small and mainly governed by diffusion [15].

The profiles of potential and ion concentrations in the central cross-section are shown in Fig. 4 for different values of surface potential $\varphi_{s}$. When the latter is small, the profiles show weak dependence on the radial coordinate $r$. With increasing $\varphi_{s}$, pronounced radial variations are observed. The counter-ions are enriched, while the co-ions are depleted in the pore. The steric effect results in the saturation of counter-ion concentration near the pore wall. The maximum concentration can be found from the expression for volume fraction of ions $\nu=C a^{3} N_{A}$, where $C$ is the ion concentration and $a$ is the ion diameter. If we put $\nu=1, C=C_{+}, a=a_{+}$, then $C_{+}^{\max }=\left(a_{+}^{3} N_{A}\right)^{-1}$. For $\mathrm{K}^{+}$ions, it leads to $C_{+}^{\max }=5778 \mathrm{~mol} / \mathrm{m}^{3}$, which is attained at the pore wall for $\varphi_{s}=-0.30 \mathrm{~V}$, see Fig. 4 (b).
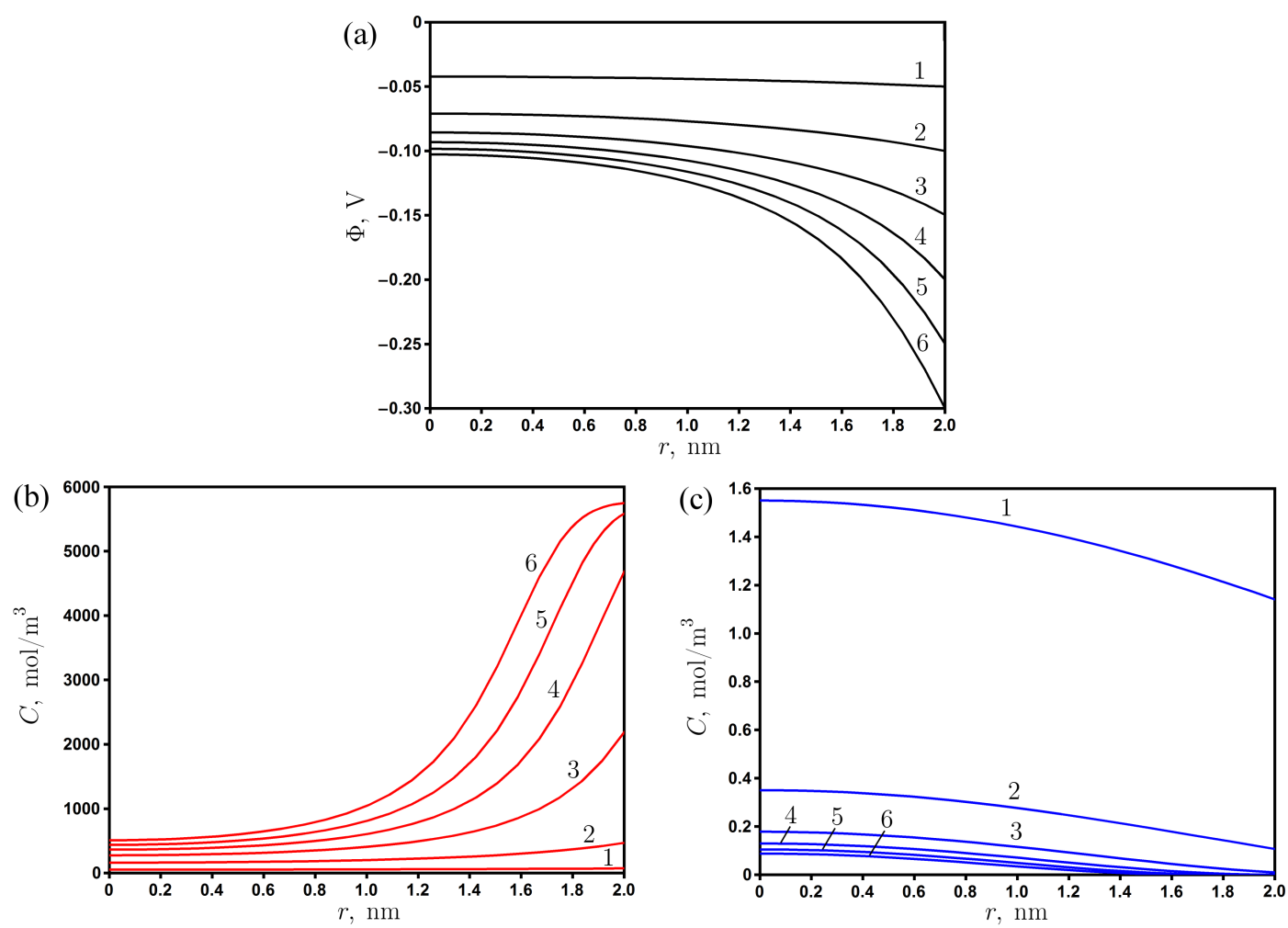

Fig. 4. The profiles of potential (a), concentrations of positive (b) and negative (c) ions in the cross-section $z=L_{p} / 2$ for different values of $\varphi_{s} .1:-0.05 \mathrm{~V}, 2:-0.10 \mathrm{~V}, 3:-0.15 \mathrm{~V}, 4$ : $-0.20 \mathrm{~V}, 5:-0.25 \mathrm{~V}, 6:-0.30 \mathrm{~V}$ 
(a)

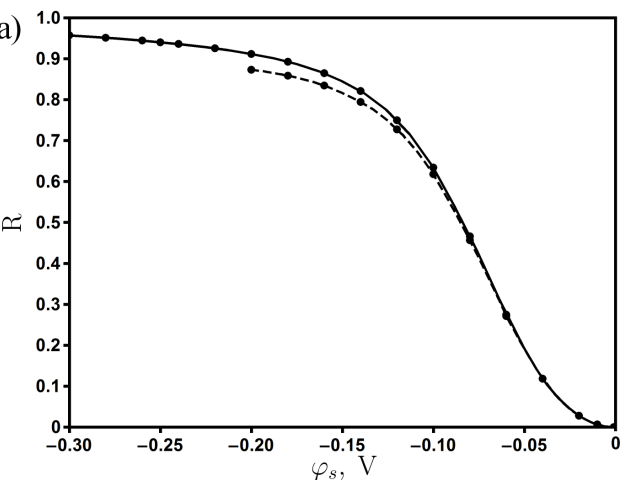

(b)

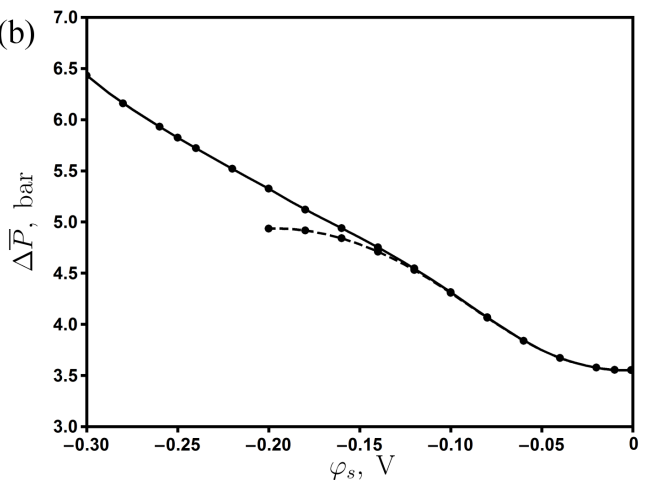

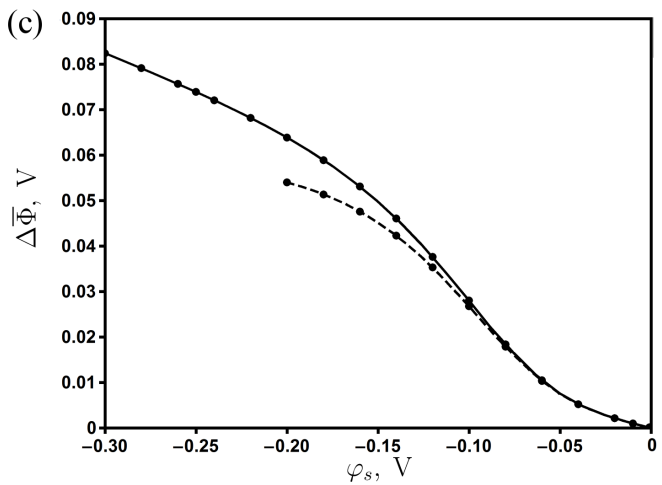

Fig. 5. The dependence of rejection (a), pressure drop (b), and membrane potential (c) on the surface potential $\varphi_{s}$. The calculated cases are shown by dots. Finite size ions (solid line), point-like ions (dashed line)

Consider now the dependence of rejection, pressure drop and membrane potential on the applied wall potential $\varphi_{s}$. The results are shown in Fig. 5. When $\varphi_{s}=0$, there are no counterion enrichment and co-ion depletion in the pore. In this case, $C^{L}=C^{R}$, so the rejection $\mathrm{R}$ and membrane potential $\Delta \bar{\Phi}$ are zero, while the pressure drop corresponds to the hydrodynamic pressure drop of Poiseuille flow. The increase of wall potential by absolute value results in the larger counter-ion enrichment and co-ion depletion, which in turn leads to the increase of rejection and membrane potential. The required pressure drop is also increased due to osmotic contribution. At small values of $\varphi_{s}$, the results for finite size and point-like ions coincide, but the deviation between these results becomes noticeable with increasing $\varphi_{s}$. For finite size ions, the calculations provide larger values of considered quantities. When $\varphi_{s}<-0.20$, the case of point-like ions is not calculated since the resulting counter-ion concentration exhibits nonphysical growth near the wall, see Fig. 2 (c). Thus, taking into account the steric effect allows to calculate the characteristics of nanofiltration process in a wider range of applied wall potentials.

\section{Conclusion}

In this paper, the pressure-driven electrolyte transport through nanofiltration membrane pores with specified wall potential has been investigated theoretically. A binary symmetric electrolyte is considered. The steric effect is taken into account by introducing an additional 
term to electrochemical potential, which results in the modification of Nernst-Planck equation and prevents the non-physical growth of ion concentration at large applied voltages. The twodimensional Navier-Stokes, Poisson, and modified Nernst-Planck equations are solved in a high aspect ratio nanopore connecting two reservoirs with a larger diameter. The Slotboom variables are introduced to simplify the equations and speed up the convergence rate.

The calculations are performed for potassium chloride aqueous solution. It is found that in the case of point-like ions, the non-physical rise of counter-ion concentration is observed near the pore wall at large applied voltages. When finite ion size is taken into account, the concentration of counter-ions decreases significantly and saturates to the maximum value. It leads to lower osmotic pressure jump and larger magnitude of potential in the pore. The stronger co-ion depletion observed for finite size ions results in the increase of salt rejection, membrane potential, and required pressure drop. The deviations between these characteristics for point-like and finite size ions become noticeable with increasing the wall potential. Taking into account the steric effect allows to calculate the characteristics of nanofiltration process in much wider range of applied voltages.

This work is supported the Russian Science Foundation, project 15-19-1001\%.

\section{References}

[1] H.Strathmann, Introduction to membrane science, Wiley-VCH, 2011.

[2] R.W.Baker, Membrane technology and applications, John Wiley \& Sons, 2004.

[3] V.V.Volkov, B.V.Mchedlishvili, V.I.Roldugin, S.S.Ivanchev, A.B.Yaroslavtsev, Membranes and nanotechnologies, Nanotechnologies in Russia, 3(2008), 656-687.

[4] M.Nishizawa, V.P.Menon, C.R.Martin, Metal nanotubule membranes with electrochemically switchable ion-transport selectivity, Science, 268(1995), 700-702.

[5] M.S.Kang, C.R.Martin, Investigations of potential-dependent fluxes of ionic permeates in gold nanotubule membranes prepared via the template method, Langmuir, 17(2001), $2753-2759$.

[6] M.W.Verbrugge, P.N.Pintauro, Transport models for ion-exchange membranes, Modern Asp. Electrochem., 19(1989), 1-67.

[7] V.V.Nikonenko, A.B.Yaroslavtsev, G.Pourcelly, Ion transfer in and through charged membranes: structure, properties, and theory, Chapter 9 in "Ionic interactions in natural and synthetic macromolecules", John Wiley \& Sons, 2012.

[8] T.Tsuru, S.Nakao, S.Kimura. Calculation of ion rejection by extended Nernst-Planck equation with charged reverse osmosis membranes for single and mixed electrolyte solutions, $J$. Chem. Eng. Jpn., 24(1991), 511-517.

[9] W.R.Bowen, H.Mukhtar, Characterisation and prediction of separation performance of nanofiltration membranes, J. Membr. Sci., 112(1996), 263-274.

[10] S.Bhattacharjee, J.C.Hen, M.Elimelech, Coupled model of concentration polarization and pore transport in crossflow nanofiltration, AIChE J., 47(2001), 2733-2745. 
[11] S.Bandini, D.Vezzani, Nanofiltration modeling: the role of dielectric exclusion in membrane characterization, Chem. Eng. Science, 58(2003), 3303-3326.

[12] R.J.Gross, J.F.Osterle, Membrane transport characteristics of ultrafine capillaries, J. Chem. Phys., 49(1968), 228-234.

[13] G.B.Westermann-Clark, J.L.Anderson, Experimental verification of the space-charge model for electrokinetics in charged microporous membranes, J. Electrochem. Soc., 130(1983), 839-847.

[14] X.L.Wang, T.Tsuru, S.I.Nakao, S.Kimura, Electrolyte transport through nanofiltration membranes by the space-charge model and the comparison with Teorell-Meyer-Sievers model, J. Membr. Sci., 103(1995), 117-133.

[15] I.I.Ryzhkov, A.V.Minakov, Theoretical study of electrolyte transport in nanofiltration membranes with constant surface potential/charge density, J. Membr. Sci., 520(2016), 515-528.

[16] P.B.Peters, R.van Roij, M.Z.Bazant, P.M.Biesheuvel, Analysis of electrolyte transport through charged nanopores, Phys. Rev. E, 93(2016), 053108.

[17] I.Borukhov, D.Andelman, H.Orland, Steric effects in electrolytes: A modified PoissonBoltzmann equation, Phys. Rev. Lett., 79(1997), 435-438.

[18] J.J.Bikerman, Structure and capacity of the electrical double layer, Philos. Mag., 33(1942), 384-397.

[19] M.S.Kilic, M.Z.Bazant, A.Ajdari, Steric effects in the dynamics of electrolytes at large applied voltages. I. Double-layer charging, Phys. Rev. E, 75(2007), 021502.

[20] M.S.Kilic, M.Z.Bazant, A.Ajdari, Steric effects in the dynamics of eletrolytes at large applied voltages. II. Modified Poisson-Nernst-Planck equations, Phys. Rev. E, 75(2007), 021503.

[21] M.Z.Bazant, M.S.Kilic, B.D.Storey, A.Ajdari, Towards an understanding of induced-charge electrokinetics at large applied voltages in concentrated solutions, Adv. Colloid Interface Sci., 152(2009), 48-88.

[22] J.Cervera, J.A.Manzanares, S.Mafé, Ion size effects on the current efficiency of narrow charged pores, J. Membr. Sci., 191(2011), 179-187.

[23] J.Cervera, V.García-Morales, J.Pellicer, Ion size effects on the electrokinetic flow in nanoporous membranes caused by concentration gradients, J. Phys. Chem. B, 107(2003), 8300-8309.

[24] H.Wang, A.Thiele, L.Pilon, Simulations of cyclic voltammetry for electric double layers in asymmetric electrolytes: A generalized modified Poisson-Nernst-Planck model, J. Phys. Chem. C, 117(2013), 18286-18297.

[25] B.Lu, Y.C.Zhou, Poisson-Nernst-Planck equations for simulating biomolecular diffusionreaction processes II: Size effects on ionic distributions and diffusion-reaction rates, Biophys. J., 100(2011), 2475-2485.

[26] E.L.Cussler, Diffusion mass transfer in fluid systems, Cambridge University Press, 2007. 
[27] B.Lu, M.J.Holst, J.A.McCammon, Y.C.Zhou, Poisson-Nernst-Planck equations for simulating biomolecular diffusion-reaction processes I: Finite element solutions, J. Comp. Phys., 229(2010), 6979-6994.

[28] T.J.Chung, Computational fluid dynamics, Cambridge University Press, 2010.

\section{Влияние конечного размера ионов на транспорт электролита в нанофильтрационных мембранах}

\section{Илья И. Рыжков}

Институт вычислительного моделирования СО РАН Академгородок, 50/44, Красноярск, 660036

Россия

Андрей В. Минаков

Институт инженерной физики и радиоэлектроники Сибирский федеральный университет Свободный, 79, Красноярск, 660041

Россия

Проведено теоретическое исследование транспорта электролита через нанофилътрационную мембрану под действием разности давлений. Стенки пор являются проводящими с заданным потенциалом. Учитывается конечный размер ионов за счет введения дополнительного слагаемого в электрохимический потенцил. Для описания течения электролита в нанопоре, соединяющей два резервуара бо́льшего диаметра, численно решаются двумерные уравнения Навъе-Стокса, Пуассона и модифицированное уравнение Нернста-Планка. Расчеты проводятся для водного раствора хлорида калия. Показано, что в случае точечных ионов значения концентрации противоионов вблизи стенки поры не соответствуют физически допустимым при больших приложенных потенциалах. При учете конечных размеров ионов концентрация противоионов существенно уменьшается, достигая максимально возможного значения вблизи стенки. Это приводит к уменъшению осмотического скачка давления и увеличению потенциала внутри поры. Более сильное исключение коионов при учете их конечного размера вызывает увеличение задержания соли, мембранного потеницала и требуемого перепада давлений.

Ключевые слова: проводящие мембраны, перенос электролита, нанофильтрация, модифицированное уравнение Нернста-Планка, конечный размер ионов, численное моделирование. 